\title{
Transcriptome-wide analysis of alternative mRNA splicing signature in the diagnosis and prognosis of stomach adenocarcinoma
}

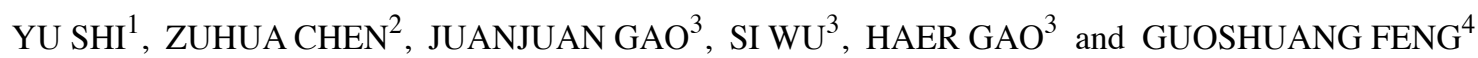 \\ ${ }^{1}$ National Clinical Research Center of Digestive Diseases, Beijing Friendship Hospital, Capital Medical University, \\ Beijing 100050; Departments of ${ }^{2}$ Gastrointestinal Oncology, and ${ }^{3}$ Cancer Epidemiology, \\ Key Laboratory of Carcinogenesis and Translational Research (Ministry of Education), \\ Peking University Cancer Hospital and Institute, Beijing 100142; ${ }^{4}$ Center for Clinical Epidemiology and \\ Evidence-based Medicine, Beijing Children's Hospital, Capital Medical University, \\ National Center for Children's Health, Beijing 100045, P.R. China
}

Received April 1, 2018; Accepted July 17, 2018

DOI: $10.3892 /$ or.2018.6623

\begin{abstract}
Alternative mRNA splicing (AS) contributes greatly to expanding the diversity and function of the proteome. Increasing evidence has suggested that dysregulation of mRNA splicing may be associated with various types of cancer. In the present study, RNA sequencing data were used to investigate alterations to the global mRNA splicing landscape of cellular genes from 452 stomach adenocarcinoma (STAD) tissues available in The Cancer Genome Atlas. Seven types of AS events, including the profiles of exon skipping events, were analyzed using SpliceSeq software. A total of $60,754 \mathrm{AS}$ events in 10,611 genes were detected, more than half of which were exon skipping events. The AS events were compared between 415 STAD tissues and 37 normal tissues, and 3,895 differentially spliced cancer-specific events were identified. In addition, the association of the AS events with the overall survival of 373 STAD patients was analyzed. Multivariate Cox regression analysis revealed that prognosis prediction models based on the AS events with clinical parameters had an excellent performance in predicting the survival of STAD patients. This study provides a comprehensive portrait
\end{abstract}

Correspondence to: Dr Guoshuang Feng, Center for Clinical Epidemiology and Evidence-based Medicine, Beijing Children's Hospital, Capital Medical University, National Center for Children's Health, South Lishi Road, Xicheng, Beijing 100045, P.R. China E-mail: glxfgsh@163.com

Abbreviations: AA, alternative acceptor site; AD, alternative donor site; AP, alternative promoter; AS, alternative mRNA splicing; AT, alternative terminator; ES, exon skipping; GC, gastric cancer; GO, Gene Ontology; ME, mutually exclusive exons; OS, overall survival; PSI, percent spliced in; RI, retained intron; STAD, stomach adenocarcinoma; TCGA, The Cancer Genome Atlas

Key words: alternative mRNA splicing, gastric cancer, cancerspecific, survival, prognosis of global changes in mRNA splicing signatures that occur in gastric cancer. These results allowed the identification of a core set of AS in gastric cancer and indicated that AS events may serve as prognostic indicators.

\section{Introduction}

Protein diversity is essential for yielding the remarkable regulatory and functional multiformity observed in human cells. A prevalent mechanism for protein diversity is the alternative processing and modification of precursor mRNA (1). Alternative mRNA splicing (AS) is a regulated process that occurs in $>95 \%$ of multi-exon human genes $(2,3)$, resulting in an enormous amount of flexibility in the regulation of gene function and protein diversity. Recent extensive genomic and functional studies have firmly established the critical role of AS in cancer (4-6). The major mechanism may be the involvement of AS in a full spectrum of oncogenic processes, including cell proliferation, apoptosis, hypoxia, angiogenesis, immune escape and metastasis $(6,7)$. In addition, a transcriptome-wide change in AS programming during epithelial-mesenchymal transition also serves an important role in cancer cell invasion and metastasis $(8,9)$.

Gastric cancer (GC) is a major global health threat and the third leading cause of cancer-associated mortality worldwide (10). In addition to DNA sequence alterations $(11,12)$, epigenetic alterations have also been extensively studied as major players in GC pathogenesis, including DNA methylation (13), histone modifications (acetylation and methylation) (14), expression of GC-associated microRNAs (15) and long non-coding RNAs (16). However, there have been relatively limited efforts to explore the mechanism of AS involved in the pathogenesis and progression of GC.

The Cancer Genome Atlas (TCGA) project provides abundant sources for the investigation of AS patterns in cancer, including data on the exon, splice and transcript isoform levels, available via the Genomic Data Commons (GDC; https://gdc.cancer.gov/). To date, systematic analyses have 
been performed to reveal the associations of the copy number variation, DNA methylation, gene expression and microRNA expression profiles with the survival of cancer patient $(17,18)$. However, the majority of RNA sequencing studies focus on identifying cancer-specific AS events $(19,20)$. A recent analysis of TCGA RNA sequencing data identified 163 cancer-specific AS events for three cancer types, among which five were found to be potentially associated with survival in breast cancer (21). There has been a lack of studies, however, that comprehensively analyze systematic cancer survival associated with AS at individual exon resolution, particularly in GC.

Recently, Armero et al (22) provided the first comprehensive portrait of global modifications in the cellular RNA splicing signatures that occur in Epstein-Barr virus (EBV)-associated GC. Since EBV infection is associated with only $10 \%$ of all GC cases reported worldwide (23), the present study aimed to systematically analyze the unclassified GC-specific AS events in the TCGA-stomach adenocarcinoma (STAD) cohort. Furthermore, survival-associated AS events were identified, and gene models with clinical parameters were constructed as prognosis predictors for GC patients.

\section{Materials and methods}

TCGA RNA sequencing data and processing. RNA sequencing read counts and medical information of GC patients, namely the TCGA-STAD cohort, were downloaded from TCGA data portal (https://tcga-data.nci.nih.gov/). The TCGA dataset provided the number of RNA sequencing read counts on the splice junctions as part of the level 3 RNA sequencing data. As shown in Fig. 1, there are seven types of splice events, as follows: Exon skipping (ES), mutually exclusive exons (ME), retained intron (RI), alternative promoter (AP), alternative terminator (AT), alternative donor site (AD) and alternative acceptor site (AA). For each ES event defined in the Ensembl annotations, the read counts were identified for exon-inclusion and skipping splice junctions.

Cancer specific mRNA splice variations analysis. SpliceSeq tool (version 2.0) (http://bioinformatics.mdanderson.org/main/ SpliceSeq:Methods) was applied to analyze the mRNA splicing patterns of TCGA-STAD samples (24), which started with a reference model for each gene constructed from all the protein-coding transcripts in the Ensembl database. The percent spliced in (PSI) index indicates how efficiently sequences of interest are spliced into transcripts, with values ranging between 0 and 1 (25). The PSI values were calculated for the seven types of AS events.

To identify possibly abnormal AS events in GC, comparisons of the PSI values were conducted between GC and normal samples (415 STAD tumors vs. 37 normal controls). Student's t-test, followed by an adjustment of the P-value (Benjamini-Hochberg method for multiple comparisons), was applied, and $\mathrm{P}<0.001$ was considered to denote a statistically significant difference.

UpSet plot, a novel visualization technique for the quantitative analysis of interactive sets (26), was also used to analyze the intersections between seven types of AS in GC.

Survival analysis. Only 373 GC patients with an overall survival (OS) of $\geq 30$ days were enrolled in the survival
Table I. Clinical parameters of patients in the stomach adenocarcinoma cohort obtained from The Cancer Genome Atlas $(n=373)$.

\begin{tabular}{|c|c|}
\hline Parameter & Value \\
\hline Age $(\text { years })^{\mathrm{a}}$ & $67(30-90)$ \\
\hline Sex (male) & 245 \\
\hline \multicolumn{2}{|l|}{ T stage } \\
\hline $\mathrm{T} 1$ & 18 \\
\hline $\mathrm{T} 2$ & 82 \\
\hline T3 & 169 \\
\hline $\mathrm{T} 4$ & 100 \\
\hline $\mathrm{TX}$ & 4 \\
\hline \multicolumn{2}{|l|}{ N stage ${ }^{b}$} \\
\hline N0 & 111 \\
\hline N1 & 105 \\
\hline $\mathrm{N} 2$ & 72 \\
\hline N3 & 75 \\
\hline NX & 9 \\
\hline \multicolumn{2}{|l|}{ M stage } \\
\hline M0 & 338 \\
\hline M1 & 22 \\
\hline MX & 13 \\
\hline \multicolumn{2}{|l|}{$\mathrm{TNM}_{\text {stage }}{ }^{\mathrm{c}}$} \\
\hline I & 50 \\
\hline II & 118 \\
\hline III & 157 \\
\hline IV & 36 \\
\hline
\end{tabular}

${ }^{\mathrm{a}}$ Age data were not available for 6 individuals; ${ }^{\mathrm{b}}$ pathologic $\mathrm{N}$ stage was not available in 1 case; ${ }^{c}$ pathologic TNM stage was not available for 12 individuals. Age is reported as the median (range).

analysis. Clinical covariates contained age, sex and the pathologic T stage, $\mathrm{N}$ stage, $\mathrm{M}$ stage and TNM stage (27), as characterized by TCGA consortium (summarized in Table I). For each AS events, GC patients were divided into two groups using the median as a cutoff value. The association between AS events and OS were evaluated by univariate Cox regression analysis. In addition, multivariate Cox regression was conducted to remove any genes that may not be independent factors in the prognostic models. Following the construction of each multivariate model, a high-risk status for GC-related death was defined when the right side of the Cox regression equation was $>0$, while low risk was defined when the value in the right side of the equation was $<0$. Based on the definition of high and low risk, survival curves were constructed to evaluate the performance of prognostic models.

Gene Ontology (GO) analysis. GO enrichment analysis (https://david.ncifcrf.gov/) was conducted to explore the functions of differentially spliced genes identified in the present study. Furthermore, the Kyoto Encyclopedia of Genes and Genomes (KEGG) database (http://www.genome.ad.jp/kegg/) was used to assess the potential functions of these target genes 


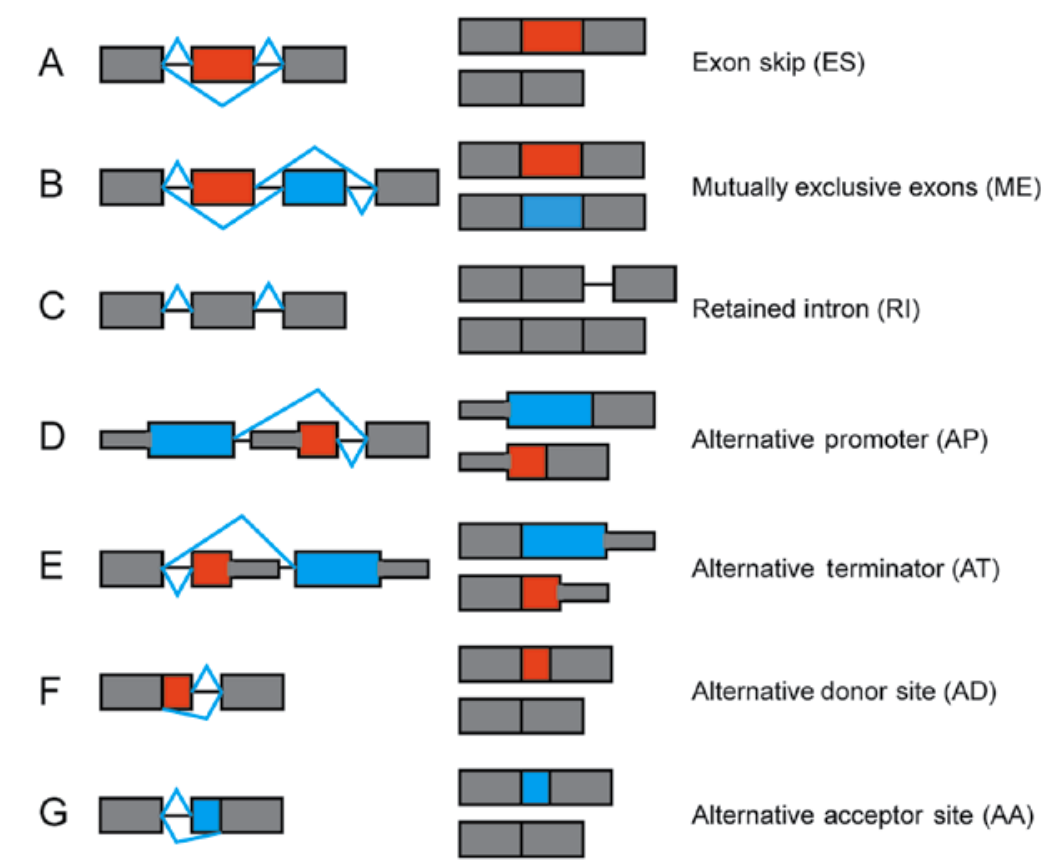

Figure 1. Illustration of the seven types of alternative splicing in the present study, including: (A) Exon skipping; (B) mutually exclusive exons; (C) retained intron; (D) alternative promoter; (E) alternative terminator; (F) alternative donor site; and (G) alternative acceptor site.

in pathways. Fisher's exact test was used to determine the GO category and GO annotation list.

Statistical analysis. All statistical analyses were performed using R/Bioconductor software (version 3.4.1; https://www. bioconductor.org/). All statistical tests were two-sided, and $\mathrm{P}=0.05$ was considered as the threshold for a statistically significant difference, unless otherwise stated.

\section{Results}

Integrated mRNA splice variant profile in the TCGA-STAD cohort. Integrated mRNA splice variant profiles were explored in depth for 452 tissues in the STAD cohort. In total, 60,754 mRNA splice variants were detected in 10,611 genes, comprised of 31,730 ES events in 6,973 genes, $226 \mathrm{ME}$ events in 219 genes, 2,944 RI events in 1,956 genes, 10,005 AP events in 4,025 genes, 8,393 AT events in 3,666 genes, 3,450 AD events in 2,401 genes and 4,006 AA events in 2,799 genes (Fig. 2). The findings of the current study indicated that AS may be universal in human multi-exon genes. Furthermore, the results demonstrated that over half of the mRNA splice variants were ES variants.

Analysis of cancer-specific mRNA splice variants. To discern any differences in mRNA splice variants, the PSI values of mRNA splice variants in STAD tumor tissues were compared with those in normal tissues (Fig. 2). Since only $7 \mathrm{ME}$ variants in 7 genes were detected to be differentially spliced in GC tissues compared with normal tissues, the study focused on the other six modes of AS for a more detailed analysis. For instance, 2,717 ES variants that belong to 402 genes were found to be differentially spliced ( $\mathrm{P}<0.001$; fold change $>2$ ). Thus, on average, a gene may have more than one cancer-specific ES event, indicating a diverse involvement of ES in GC.
The top ranked GC-specific splice genes were labeled in the volcano plots, with the exception of ME with only 7 variants (Fig. 3). Furthermore, it was observed that there were a few genes that had more than one type of mRNA splice variant and were differentially spliced in GC. The intersection of gene sets is visualized in Fig. 4. For instance, RPS6 and UBE2C had four types of variants (ES, RI, AD and AA) that were differentially spliced in GC. In addition, the ES, AT, $\mathrm{AD}$ and $\mathrm{AA}$ variants in gene RPS3A were also differentially spliced.

GO and KEGG pathway analysis of cancer-specific differentially spliced genes. Through GO analysis, genes were organized into hierarchical categories to uncover gene regulatory networks on the basis of biological process (excluding the ME events). It was observed that, among these differentially spliced genes, 1,320 are involved in biological processes, 1,380 in cellular components and 1,149 in molecular functions (Fig 5A-C).

To further specify and identify target mRNAs (excluding the ME events), significant pathways of differentially spliced mRNAs were analyzed using the KEGG database (Fig. 5D). This analysis revealed that GC-specific ES genes were focused in six pathways, including Salmonella infection, bacterial invasion of epithelial cells, shigellosis, pathogenic Escherichia coli infection, ribosome and adrenergic signaling in cardiomyocytes. Ribosome was the only significantly enriched network for AA, AD, AT, ES and RI variants.

Association of OS with $\mathrm{mRNA}$ splice variants in the TCGA$S T A D$ cohort. Univariate survival tests were first conducted to assess the correlation between clinical parameters and OS in the STAD cohort. The age, $\mathrm{T}$ stage, $\mathrm{N}$ stage, $\mathrm{M}$ stage and TNM stage were significantly associated with OS. Next, univariate survival analysis for OS was conducted using the integrated 
Table II. List of survival-associated splicing factors of genes in multivariate Cox analysis.

Alternative splicing
Genes

\begin{tabular}{ll}
\hline AA & CBX7, DHPS, DYNLL1, LMO7, MORF4L2, RPS21, SCYL1, SHQ1, SMUG1, SUN2, TCTN1, TNIP1 \\
AD & BMP4, C1QC, DAGLB, E2F5, GK5, IRF9, LYRM9, MARVELD3, MGME1, MT1F, NFATC1, \\
& NOB1, RALGPS1, TAF1D, TXNDC9, WDR20 \\
AP & ALDOA, CCDC64B, CCND3, CLIP3, CTBP2, DALRD3, DCTN1, HOXB3, IL1R1, KDM2B, KIAA1671, \\
& MICAL2, NEDD1, NFATC2, NNT, NR2F2, PKM, PLAGL1, RTN4, SGIP1, TCF4, TNFAIP8L1, ZNF544 \\
AT & ABCB5, ABCC5, ACAD9, C1orf52, CD300LG, CPED1, DYNLL1, EDA, GHR, IL7R, MRPL30, NOX4, \\
& PRTG, REXO2, RMDN2, SLC35G1, SMG8, SPINK5, TSTD2, UQCC1, WNT9B, ZBTB8OS, \\
& ZNF407, ZNF680, ZNF846 \\
ES & ABI1, ARHGAP4, C14orf80, CD44, EML2, FSTL1, GGA3, GRIPAP1, HPGD, LDB2, MAP4, \\
& MAPKBP1, MEF2B, \\
& NCOA7, NFATC3, P4HA2, PLAGL1, RHOT1, RQCD1, SAE1, SEC16A, SEC31A, SLC27A2, \\
UE & G3BP1, GRB10, H2AFY, ZDHHC16, ZNF140 \\
RI & BICD2, BRWD1, C18orf21, COPZ1, CYHR1, FAU, FOS, METTL3, SMUG1, UBE2V1 \\
\hline
\end{tabular}

AA, alternative acceptor site; AD, alternative donor site; AP, alternative promoter; AT, alternative terminator; ES, exon skipping; ME, mutually exclusive exons; RI, retained intron.

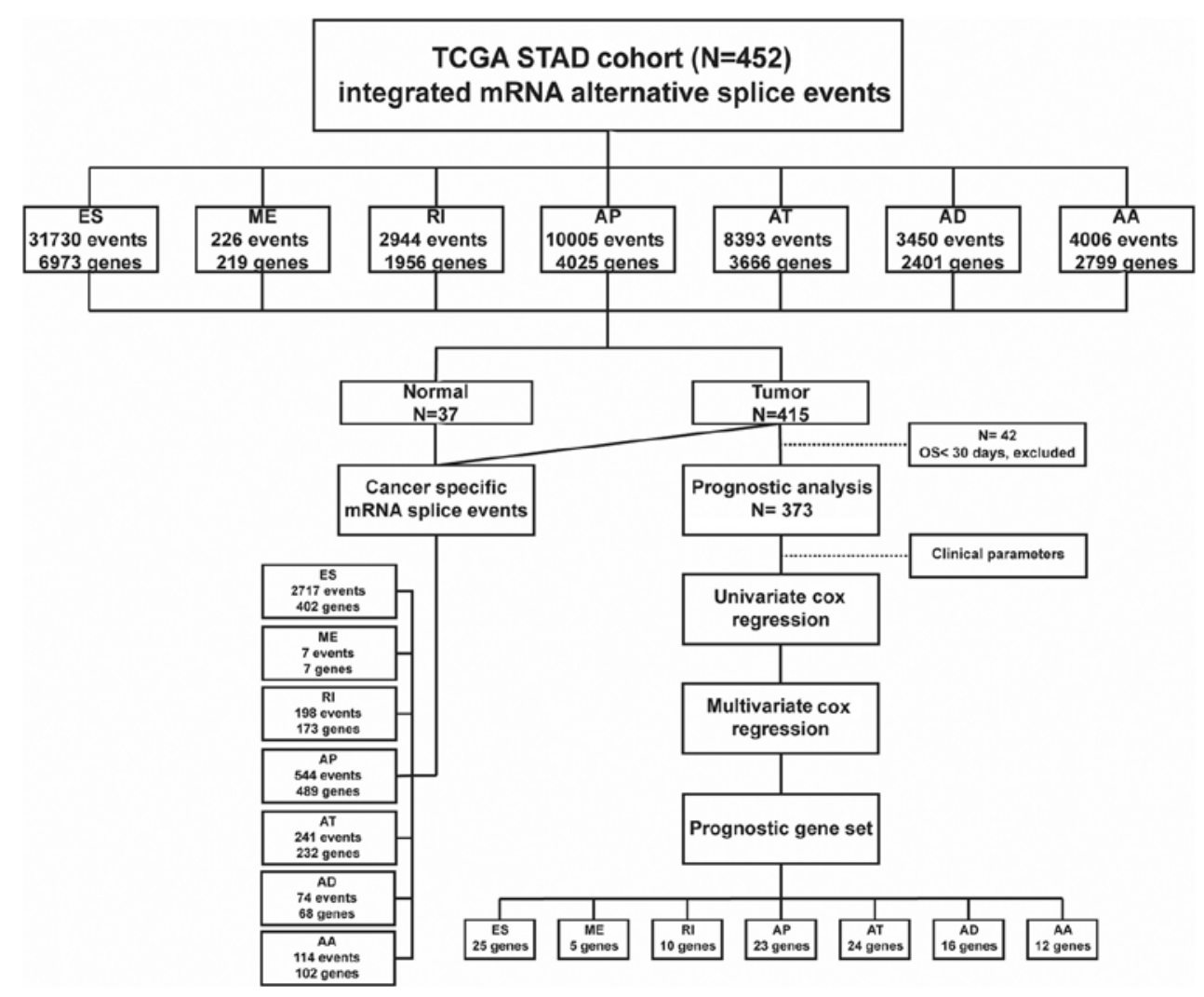

Figure 2. Flow chart of the study design. AA, alternativee acceptor site; AD, alternative donor site; AP, alternativee promoter; AT, alternative terminator; ES, exon skipping; ME, mutually exclusive exons; RI, retained intron; TCGA, The Cancer Genome Atlas; STAD, stomach adenocarcinoma.

mRNA splice variants profiles in the STAD cohort. The univariate survival analyses identified that 1,168 ES, $16 \mathrm{ME}$, 138 RI, 675 AP, 434 AT, 186 AD and 170 AA events were statistically associated with OS. The top 20 genes that were most significantly associated with survival in the six types of AS are presented in Fig. 6 (excluding the ME events). 

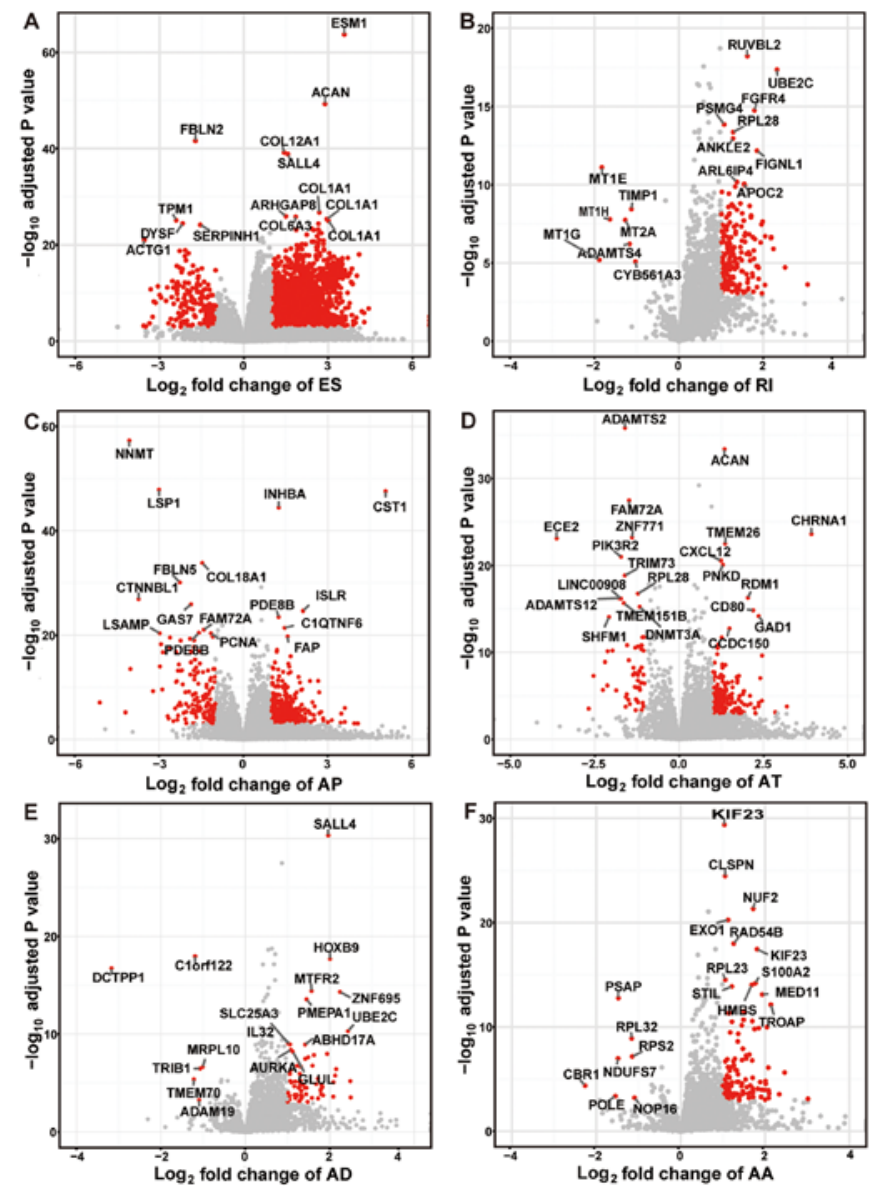

Figure 3. Volcano plots for the six types of alternative splicing with top ranked cancer-specific splice genes. (A) ES, (B) RI, (C) AP, (D) AT, (E) AD and (F) AA event plots are shown. The y-axis represents the negative $\log _{10}$ $\mathrm{P}$-values, while the $\mathrm{x}$-axis represents the $\log _{2}$ of the fold change for every type of alternative splicing. The red dots indicate that alternative splicing variants were found to be significantly differentially spliced between GC tissues and normal tissues. The gene names of top ranked cancer-specific splice variants are shown. AA, alternative acceptor site; AD, alternative donor site; AP, alternative promoter; AT, alternative terminator; ES, exon skipping; RI, retained intron.

Integrated STAD risk predictor: Prognostic gene sets. Subsequent to determining the candidate genes across the seven types of mRNA splice variants, multivariate Cox regression was separately applied to the candidate gene sets for each of the six types of AS (Fig. 2). The ES variants in 25 genes were found to be independent prognostic factors in the survival of STAD patients. Notably, MAPKBP1 was one of the most significant independent prognostic factors [hazard ratio (HR), 116.77; 95\% confidence interval (95\% CI), 15.66-870.87; $\mathrm{P}<0.001]$. The age, $\mathrm{T}$ stage, $\mathrm{N}$ stage and $\mathrm{M}$ stage were also independent prognostic factors in the models. Similarly, numerous genes were identified as independent prognostic factors in the other six types of mRNA splice variants. The specific AS events of genes involved in the final models are listed in Table II.

Based on the aforementioned prognostic models, Kaplan-Meier survival plots were generated to test the performance of these models as shown in Fig. 7 (excluding ME). All six mRNA splice variant prognostic models were significantly associated with the OS of GC patients (all $\mathrm{P}<0.05$ ).

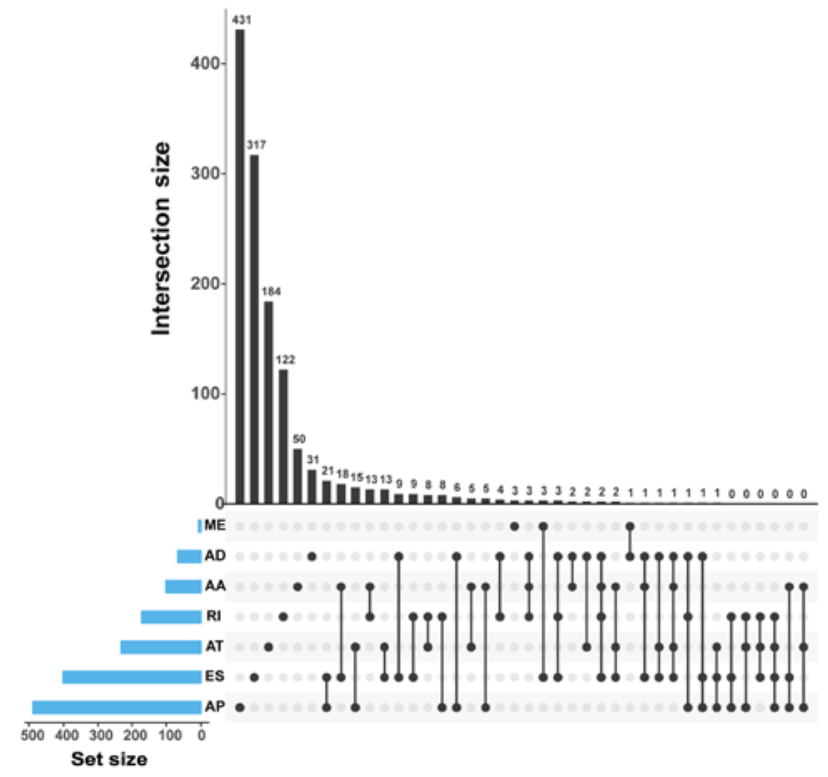

Figure 4. UpSet plots in STAD, showing the interactions between the seven types of GC-specific alternative splicing events in STAD. One gene may have up to four types of alternative splicing that are differentially spliced in STAD. AA, alternative acceptor site; AD, alternative donor site; AP, alternative promoter; AT, alternative terminator; ES, exon skipping; ME, mutually exclusive exons; RI, retained intron; STAD, stomach adenocarcinoma.

Furthermore, the prediction models constructed for the splice variants ES, AP, AT, AD and AA were good, since evident gaps between the curves of high-risk and low-risk patients were observed.

\section{Discussion}

AS generally allows cells to generate substantial mRNA and protein isoforms with diverse regulatory and functional properties, which may also facilitate the survival, proliferation and metastasis of cancer cells $(6,7)$. The present study reported a comprehensive transcriptome-wide analysis of the AS profiling landscape for GC, and identified a significant association between AS and the GC clinical outcome. Through an analysis of the RNA-Seq data of individuals in the TCGA-STAD cohort, a total of 60,754 mRNA splice variants were detected in 10,611 genes. Recently, Tsai et al (21) reported that there were individually 65,152, 70,342 and 70,637 AS events in breast, liver and lung cancer, respectively, all of which have a slightly higher number of AS events compared with that reported for GC in the present study.

Systematic identification and analysis of cancer-specific AS events was also performed in the current study, using TCGASTAD data. The results revealed that there are thousands of AS events significantly associated with GC, involving multiple genes whose splicing is known to serve a critical role in cancer development, such as CD44 (28). Several studies have demonstrated that polymorphisms in CD44 are associated with a risk for several types of cancer, including breast cancer (29), colorectal cancer (30) and GC (31). In the present study, CD44 was differentially spliced between GC and normal tissues, and the main splicing modes were ES and AA. It was also observed that one type of ES events for CD44 was negatively associated with the survival in GC. However, the AS mechanisms for 

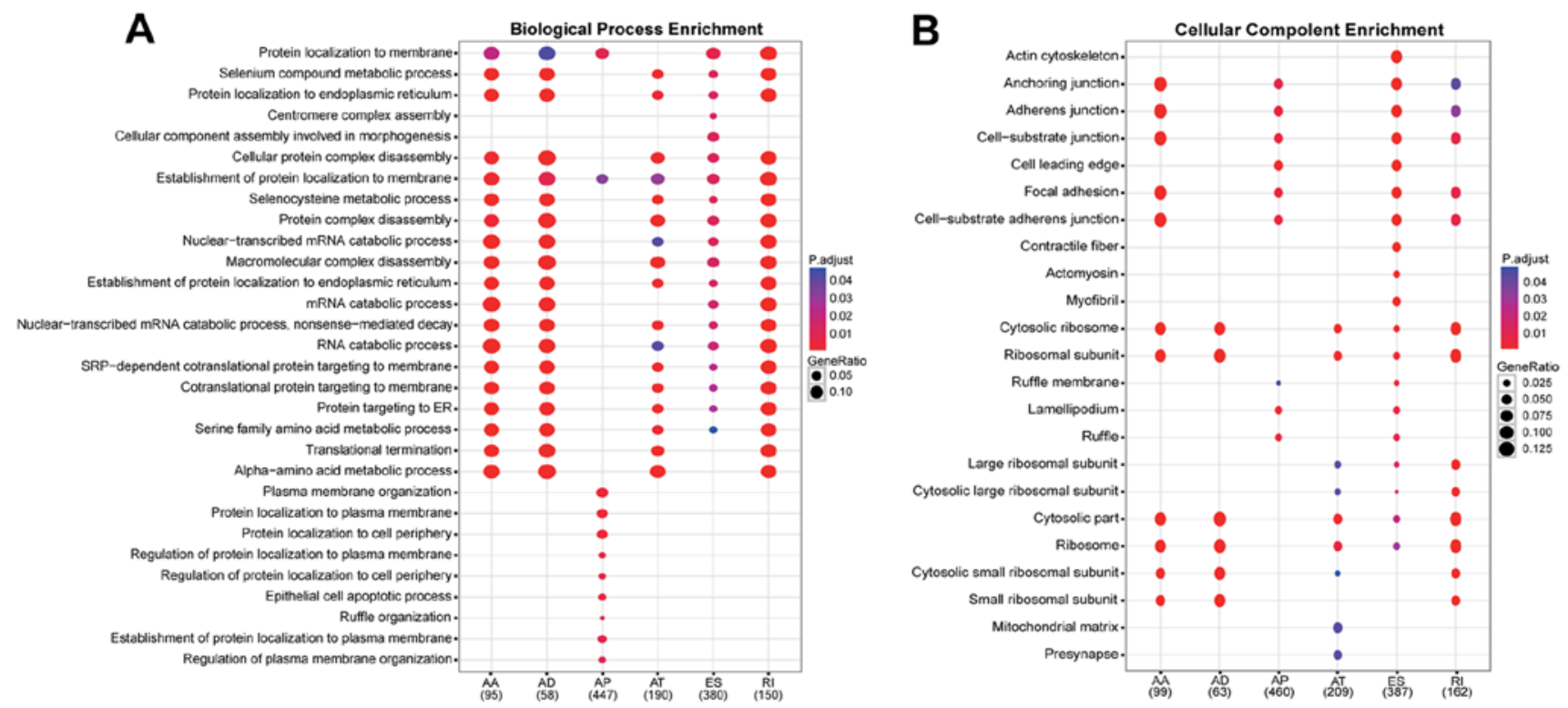

C

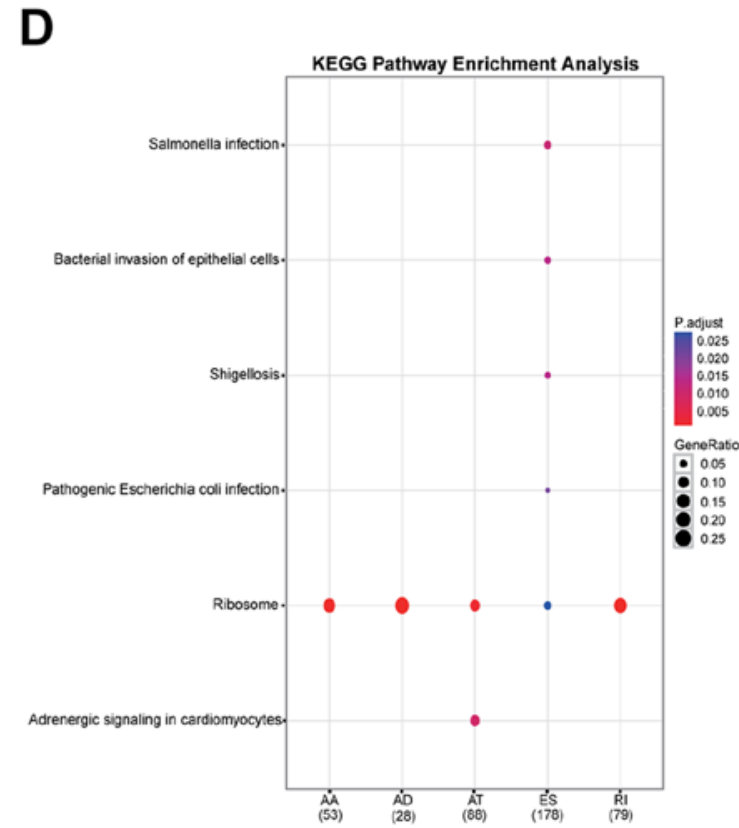

Figure 5. Go and KEGG analysis of differentially spliced genes. (A) Biological process. (B) Cellular components. (C) Molecular functions. (D) KEGG pathway.

CD44 have yet to be explicitly defined, and the role of different CD44 spliced isoforms in the development and metastasis of GC should be more clearly elucidated in future studies. Conversely, several AS events of genes with well-known roles in cancer development were not identified by our procedure, possibly due to the highly stringent inclusion criteria applied in the present study. For instance, GC-specific AS genes in the present study did not include Bcl-xL, whose splicing is known to control cell apoptosis in multiple tumors (32). In addition, CDH1 was also not identified as a GC-specific AS gene, yet CDH1 exon 11 skipping has been reported in leukemia cells, and head and neck cancer cells $(33,34)$, while a fragment lacking the final 83 base pairs of exon 8 in CDH1 was frequently detected in Chinese GC patients (35). The fold change of Bcl-xL and CDH1 was not, however, large enough to pass the significance thresholds in the present analysis. It is expected that additional cancer-specific genes could be identified if these criteria are relaxed. Furthermore, the P-value of
AS events was required to be $<0.001$, which may cause certain uncommon events to be omitted in the current study.

Notably, RPS6 and UBE2C were found to have four splicing modes (ES, RI, AD and AA) of variants that were differentially spliced in GC. RPS6, a component of the 40S ribosomal subunit, is regarded to be involved in the translation of specific mRNAs, as well as a regulator of cellular metabolisms, survival and proliferation (36). Several studies have reported the overexpression and activation of RPS6 in esophageal squamous cell carcinoma (37), lung cancer (38) and breast cancer (39). Furthermore, the present study demonstrated that increased ES events in RPS6 were associated with poor prognosis. UBE2C encodes a member of the E2 ubiquitin-conjugating enzyme family, which is involved in the destruction of mitotic cyclins and cell cycle progression (40). Overexpression of UBE2C is associated with worse clinical outcomes in a number of cancer types, including GC (41). MAPKBP1, acting as a scaffold protein, facilitates 

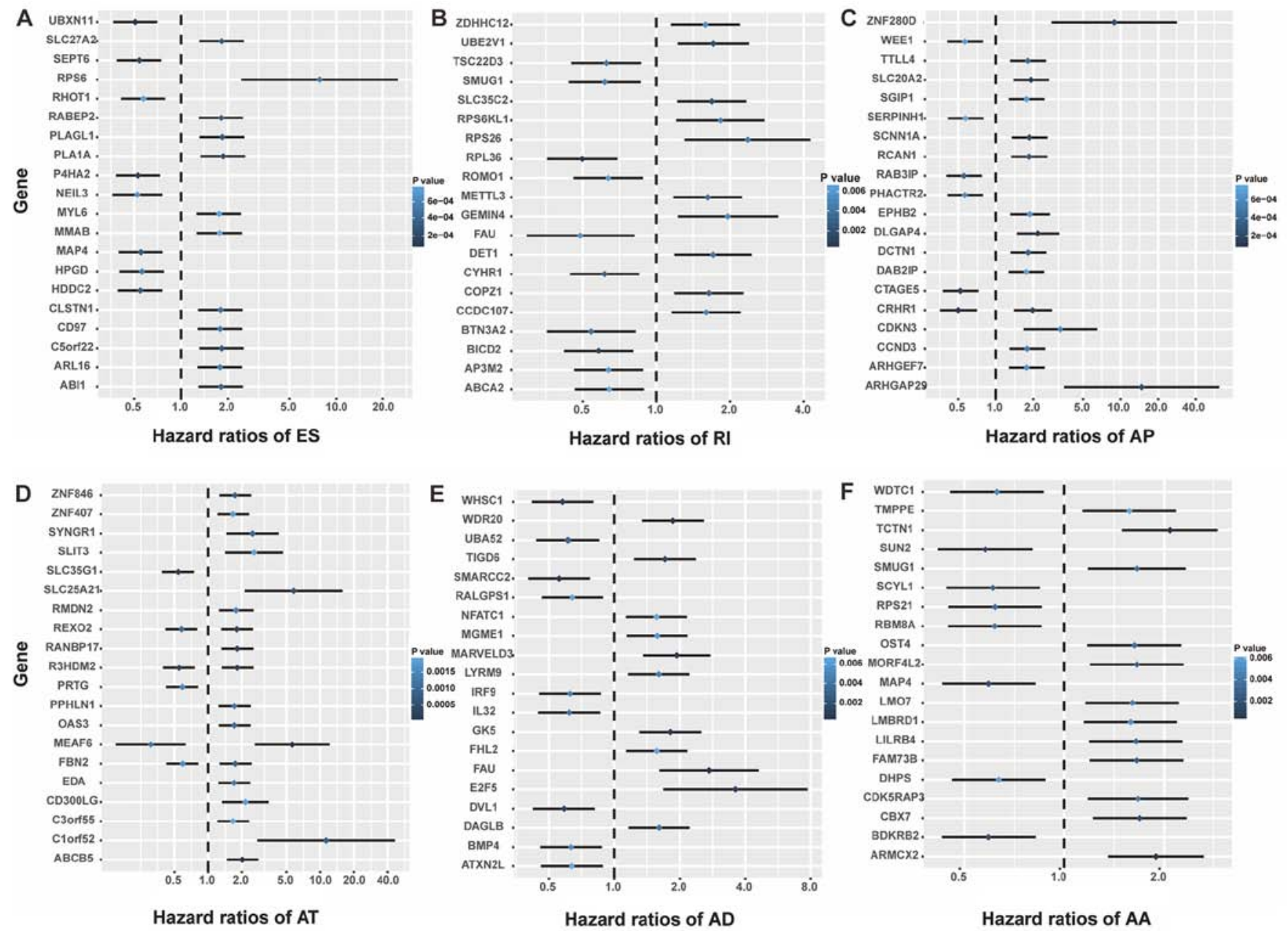

Figure 6. Forrest plots of hazard ratios for survival-associated alternative events in stomach adenocarcinoma. Hazard ratios of the top 20 genes with survivalassociated (A) ES, (B) RI, (C) AP, (D) AT, (E) AD and (F) AA events. AA, alternative acceptor site; AD, alternative donor site; AP, alternative promoter; AT, alternative terminator; ES, exon skipping; ME, mutually exclusive exons; RI, retained intron.

the polyubiquitination of TNF-receptor associated factor 2 , leading to the TGF- $\beta$-activated kinase 1-mediated activation of nuclear factor- $\kappa \mathrm{B}(42,43)$. The results of the current study also revealed that MAPKBP1 was one of the most significant independent prognostic factors. Fu et al (44) reported the prognostic relevance of MAPKBP1 expression in cytogenetically normal acute myeloid leukemia. This evidence probably suggests the functional and clinical relevance of these genes in the survival-associated AS events. While certain of these events may reflect a correlation but may not have a causal effect on the survival of GC patients, others may serve an active role in regulating the cancer cell phenotypes, cancer development and cancer prognosis. Therefore, AS events for these marked genes should be investigated further to elucidate their impact on the development and prognosis of GC.

Currently, AS has been considered to be generally regulated by multiple cis-acting splicing regulatory elements that are specifically bound by trans-acting splicing factors to enhance or inhibit the use of nearby splice sites $(45,46)$. AS events can respond not only to various signaling pathways that target splicing mechanisms, but can also be influenced by transcription factors and chromatin structure (47). Small interfering RNAs have been reported to trigger AS events in human cells through transcriptional gene silencing pathways (48). MicroRNAs can also serve an important role in the regulation of AS. For instance, miR-124 promotes neuronal differentiation by targeting the PTBP1 splicing repressor (49). Shukla et al (50) reported that DNA methylation can regulate AS in CD45 exon 5. Furthermore, the expression of numerous cellular splicing factors has been reported to be dysregulated in various human diseases. Armero et al (22) detected several changes in the expression levels and/or splicing patterns of cellular splicing factors in EBV-negative GC tissues, including CDK10, MBNL1 and RBFOX2. Notably, recent data demonstrated a key role for RBFOX2 and MBNL1, which seem to account for numerous splicing alterations in breast, lung and prostate cancer (51). The current study also identified that ES events in RBFOX2 and MBNL1 were significantly different in GC samples. Although a few known factors regulating AS events can be listed, it is likely that there are numerous other regulators awaiting identification and validation.

In conclusion, the present study generated a common set of cancer-specific and survival-associated AS events in GC. Furthermore, the results demonstrated that a core set of AS events can be potentially applied to build predictive models for the survival of GC patients with a higher accuracy. This further demonstrates the potential biological relevance and clinical utility of these identified AS events. Ultimately, certain RNA 

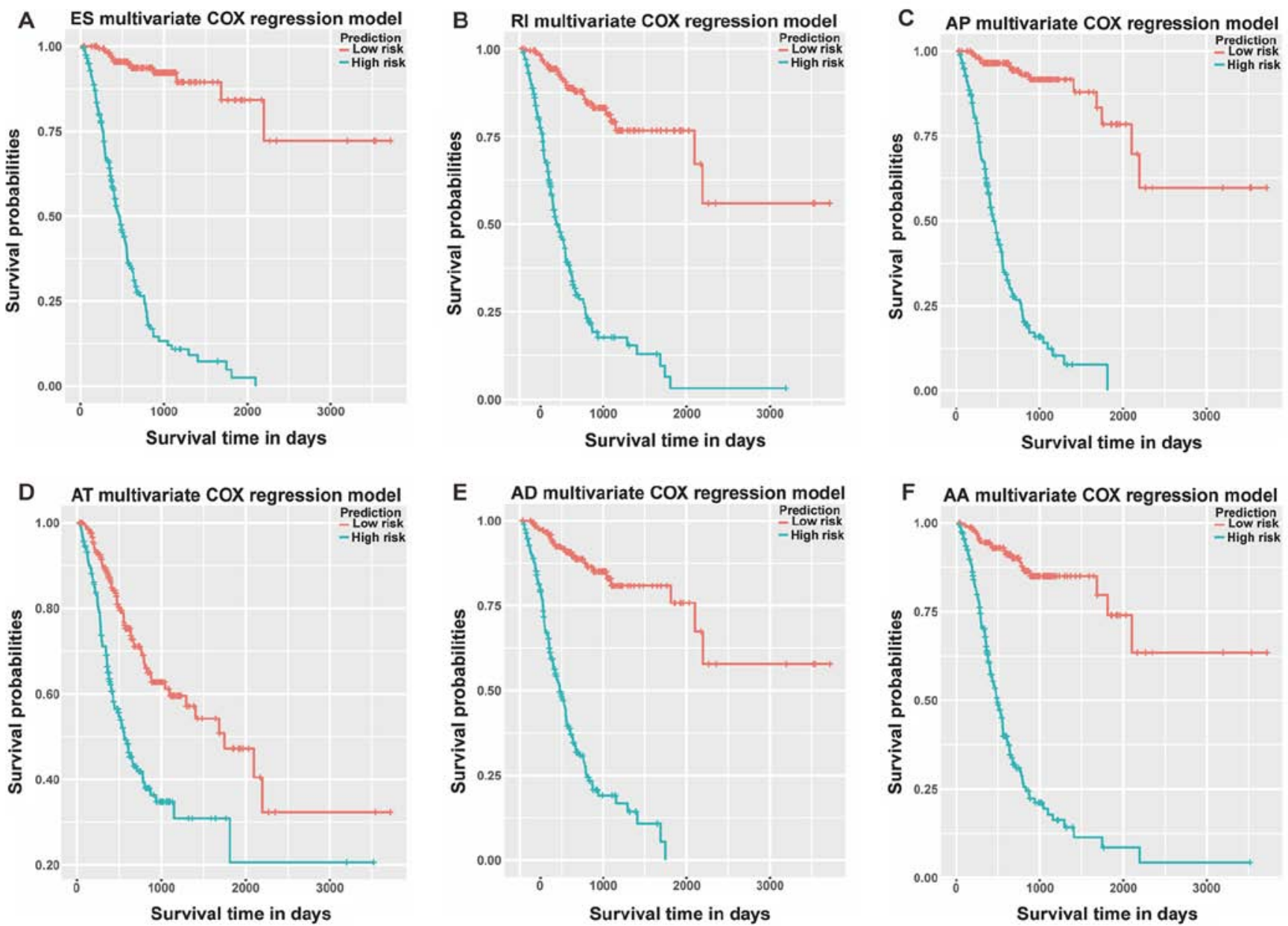

Figure 7. Kaplan-Meier prognostic predictors for gastric cancer. Prognostic predictor models were constructed according to the (A) ES, (B) RI, (C) AP, (D) AT, (E) AD and (F) AA events in stomach adenocarcinoma. AA, alternative acceptor site; AD, alternative donor site; AP, alternative promoter; AT, alternative terminator; ES, exon skipping; ME, mutually exclusive exons; RI, retained intron.

splicing isoforms may eventually be used as diagnostic or prognostic biomarkers, and even as novel therapeutic targets.

\section{Acknowledgements}

Not applicable.

\section{Funding}

No funding was received.

\section{Availability of data and materials}

The datasets used or analysed during the current study are available from the corresponding author on reasonable request.

\section{Authors' contributions}

YS and GF were involved in the design of the study. YS, JG, SW and HG conducted the statistical analysis and compiled the figures. ZC collected the original data. All authors read and approved the final manuscript and agreed to be accountable for all aspects of the work in ensuring that questions related to the accuracy or integrity of any part of the work are appropriately investigated and resolved.

\section{Ethics approval and consent to participate}

Not applicable.

\section{Patient consent for publication}

Not applicable.

\section{Competing interests}

The authors declare that they have no conflict of interest.

\section{References}

1. Nilsen TW and Graveley BR: Expansion of the eukaryotic proteome by alternative splicing. Nature 463: 457-463, 2010.

2. Wang ET, Sandberg R, Luo S, Khrebtukova I, Zhang L, Mayr C, Kingsmore SF, Schroth GP and Burge CB: Alternative isoform regulation in human tissue transcriptomes. Nature 456: 470-476, 2008.

3. Pan Q, Shai O, Lee LJ, Frey BJ and Blencowe BJ: Deep surveying of alternative splicing complexity in the human transcriptome by high-throughput sequencing. Nat Genet 40: 1413-1415, 2008.

4. Venables JP: Aberrant and alternative splicing in cancer. Cancer Res 64: 7647-7654, 2004.

5. Kim E, Goren A and Ast G: Insights into the connection between cancer and alternative splicing. Trends Genet 24: 7-10, 2008.

6. Oltean S and Bates DO: Hallmarks of alternative splicing in cancer. Oncogene 33: 5311-5318, 2014. 
7. Liu S and Cheng C: Alternative RNA splicing and cancer. Wiley Interdiscip Rev RNA 4: 547-566, 2013.

8. De Craene B and Berx G: Regulatory networks defining EMT during cancer initiation and progression. Nat Rev Cancer 13: 97-110, 2013.

9. Warzecha CC, Jiang P, Amirikian K, Dittmar KA, Lu H, Shen S, Guo W, Xing Y and Carstens RP: An ESRP-regulated splicing programme is abrogated during the epithelial-mesenchymal transition. EMBO J 29: 3286-3300, 2010.

10. Torre LA, Bray F, Siegel RL, Ferlay J, Lortet-Tieulent J and Jemal A: Global cancer statistics, 2012. CA Cancer J Clin 65: 87-108, 2015

11. Hudler P: Genetic aspects of gastric cancer instability. Sci World J 2012: 761909, 2012.

12. Tao J, Deng NT, Ramnarayanan K, Huang B, Oh HK, Leong SH, Lim SS, Tan IB, Ooi CH, Wu J, et al: CD44-SLC1A2 gene fusions in gastric cancer. Sci Transl Med 3: 77ra30, 2011.

13. Zouridis H, Deng N, Ivanova T, Zhu Y, Wong B, Huang D, Wu YH, Wu Y, Tan IB, Liem N, et al: Methylation subtypes and large-scale epigenetic alterations in gastric cancer. Sci Transl Med 4: 156ra140, 2012.

14. Calcagno DQ, Gigek CO, Chen ES, Burbano RR and Smith MA DNA and histone methylation in gastric carcinogenesis. World J Gastroenterol 19: 1182-1192, 2013.

15. Wu WK, Lee CW, Cho CH, Fan D, Wu K, Yu J and Sung JJ: MicroRNA dysregulation in gastric cancer: A new player enters the game. Oncogene 29: 5761-5771, 2010.

16. Li T, Mo X, Fu L, Xiao B and Guo J: Molecular mechanisms of long noncoding RNAs on gastric cancer. Oncotarget 7: $8601-8612,2016$

17. Ludwig JA and Weinstein JN: Biomarkers in cancer staging, prognosis and treatment selection. Nat Rev Cancer 5: 845-856, 2005 .

18. Yuan Y, Van Allen EM, Omberg L, Wagle N, Amin-Mansour A, Sokolov A, Byers LA, Xu Y, Hess KR, Diao L, et al: Assessing the clinical utility of cancer genomic and proteomic data across tumor types. Nat Biotechnol 32: 644-652, 2014.

19. Li XW, Shi BY, Yang QL, Wu J, Wu HM, Wang YF, Wu ZJ, Fan YM and Wang YP: Epigenetic regulation of CDH1 exon 8 alternative splicing in gastric cancer. BMC Cancer 15: 954, 2015.

20. Hafezi N, Ajami A, Farazmandfar T, Hosseini V, AlizadehNavaei R and Tehrani M: Increased expression of two alternative spliced variants of CD1d molecule in human gastric cancer. Iran J Immunol 12: 129-140, 2015.

21. Tsai YS, Dominguez D, Gomez SM and Wang Z: Transcriptome-wide identification and study of cancer-specific splicing events across multiple tumors. Oncotarget 6: 6825-6839, 2015.

22. Armero VE, Tremblay MP, Allaire A, Boudreault S Martenon-Brodeur C, Duval C, Durand M,Lapointe E, Thibault P, Tremblay-Létourneau $\mathrm{M}$, et al: Transcriptome-wide analysis of alternative RNA splicing events in Epstein-Barr virus-associated gastric carcinomas. PLoS One 12: e0176880, 2017.

23. Uozaki $\mathrm{H}$ and Fukayama $\mathrm{M}$ : Epstein-Barr virus and gastric carcinoma - viral carcinogenesis through epigenetic mechanisms. Int J Clin Exp Pathol 1: 198-216, 2008.

24. Ryan M, Wong WC, Brown R, Akbani R, Su X, Broom B, Melott J and Weinstein J: TCGASpliceSeq a compendium of alternative mRNA splicing in cancer. Nucleic Acids Res 44: D1018-D1022, 2016.

25. Ryan MC, Cleland J, Kim R, Wong WC and Weinstein JN: SpliceSeq: A resource for analysis and visualization of RNA-Sec data on alternative splicing and its functional impacts. Bioinformatics 28: 2385-2387, 2012.

26. Lex A, Gehlenborg N, Strobelt H, Vuillemot R and Pfister $H$ : UpSet: Visualization of intersecting sets. IEEE Trans Vis Comput Graph 20: 1983-1992, 2014.

27. Sobin LH, Gospodarowicz MK and Wittekind C (eds): TNM classification of malignant tumours. 7th edition. Wiley-Blackwell, Oxford, p310, 2009

28. Brown RL, Reinke LM, Damerow MS, Perez D, Chodosh LA, Yang J and Cheng C: CD44 splice isoform switching in human and mouse epithelium is essential for epithelial-mesenchymal transition and breast cancer progression. J Clin Invest 121 1064-1074, 2011

29. Jiang L, Deng J, Zhu X, Zheng J, You Y, Li N, Wu H, Lu J and Zhou Y: CD44 rs13347 C>T polymorphism predicts breast cancer risk and prognosis in Chinese populations. Breast Cancer Res 14: R105, 2012.
30. Wu XM, Yang HG, Zheng BA, Cao HF, Hu ZM and Wu WD: Functional genetic variations at the microRNA binding-site in the CD44 gene are associated with risk of colorectal cancer in Chinese populations. PLoS One 10: e0127557, 2015.

31. Suenaga M, Yamada S, Fuchs BC, Fujii T, Kanda M, Tanaka C, Kobayashi D, Fujiwara M, Tanabe KK and Kodera Y: CD44 single nucleotide polymorphism and isoform switching may predict gastric cancer recurrence. J Surg Oncol 112: 622-628, 2015.

32. Adams JM and Cory S: The Bcl-2 apoptotic switch in cancer development and therapy. Oncogene 26: 1324-1337, 2007.

33. Sharma S and Lichtenstein A: Aberrant splicing of the E-cadherin transcript is a novel mechanism of gene silencing in chronic lymphocytic leukemia cells. Blood 114: 4179-4185, 2009.

34. Sharma S, Liao W, Zhou X, Wong DT and Lichtenstein A: Exon 11 skipping of E-cadherin RNA downregulates its expression in head and neck cancer cells. Mol Cancer Ther 10: 1751-1759, 2011

35. Li X, Gao Y, Pan Y, Pan Y, Wang L, Xiao N, He Q, Fan Y and Wang Y: Mutation screen and RNA analysis disclose the changed splicing of the E-cadherin transcription in gastric cancer. Fam Cancer 12: 547-554, 2013

36. Ruvinsky I and Meyuhas O: Ribosomal protein S6 phosphorylation: From protein synthesis to cell size. Trends Biochem Sci 31: 342-348, 2006.

37. Kim SH, Jang YH, Chau GC, Pyo S and Um SH: Prognostic significance and function of phosphorylated ribosomal protein S6 in esophageal squamous cell carcinoma. Mod Pathol 26: 327-335, 2013.

38. Chen B, Tan Z, Gao J, Wu W, Liu L, Jin W, Cao Y, Zhao S, Zhang W, Qiu Z, et al: Hyperphosphorylation of ribosomal protein S6 predicts unfavorable clinical survival in non-small cell lung cancer. J Exp Clin Cancer Res 34: 126, 2015.

39. Akar U, Ozpolat B, Mehta K, Lopez-Berestein G, Zhang D, Ueno NT, Hortobagyi GN and Arun B: Targeting p70S6K prevented lung metastasis in a breast cancer xenograft model. Mol Cancer Ther 9: 1180-1187, 2010.

40. Pagano M: Cell cycle regulation by the ubiquitin pathway. FASEB J 11: 1067-1075, 1997.

41. Xie C, Powell C, Yao M, Wu J and Dong Q: Ubiquitin-conjugating enzyme E2C: A potential cancer biomarker. Int J Biochem Cell Biol 47: 113-117, 2014.

42. Lee SW, Han SI, Kim HH and Lee ZH: TAK1-dependent activation of AP-1 and c-Jun N-terminal kinase by receptor activator of NF-kappaB. J Biochem Mol Biol 35: 371-376, 2002.

43. Yamaguchi T, Miyashita C, Koyano S, Kanda H, Yoshioka K, Shiba T, Takamatsu $\mathrm{N}$ and Ito M: JNK-binding protein 1 regulates NF-kappaB activation through TRAF2 and TAK1. Cell Biol Int 33: 364-368, 2009.

44. Fu L, Shi J, Hu K, Wang J, Wang W and Ke X: Mitogen-activated protein kinase binding protein 1 (MAPKBP1) is an unfavorable prognostic biomarker in cytogenetically normal acute myeloid leukemia. Oncotarget 6: 8144-8154, 2015.

45. Matera AG and Wang Z: A day in the life of the spliceosome. Nat Rev Mol Cell Biol 15: 108-121, 2014.

46. Matlin AJ, Clark F and Smith CW: Understanding alternative splicing: Towards a cellular code. Nat Rev Mol Cell Biol 6: 386-398, 2005

47. Naftelberg S, Schor IE, Ast G and Kornblihtt AR: Regulation of alternative splicing through coupling with transcription and chromatin structure. Annu Rev Biochem 84: 165-198, 2015.

48. Alló M, Buggiano V, Fededa JP, Petrillo E, Schor I, de la Mata M, Agirre E, Plass M, Eyras E, Elela SA, et al: Control of alternative splicing through siRNA-mediated transcriptional gene silencing. Nat Struct Mol Biol 16: 717-724, 2009.

49. Makeyev EV, Zhang J, Carrasco MA and Maniatis T: The MicroRNA miR-124 promotes neuronal differentiation by triggering brain-specific alternative pre-mRNA splicing. Mol Cell 27: 435-448, 2007.

50. Shukla S, Kavak E, Gregory M, Imashimizu M, Shutinoski B, Kashlev M, Oberdoerffer P, Sandberg R and Oberdoerffer S: CTCF-promoted RNA polymerase II pausing links DNA methylation to splicing. Nature 479: 74-79, 2011

51. Danan-Gotthold M, Golan-Gerstl R, Eisenberg E, Meir K, Karni R and Levanon EY: Identification of recurrent regulated alternative splicing events across human solid tumors. Nucleic Acids Res 43: 5130-5144, 2015.

This work is licensed under a Creative Commons Attribution-NonCommercial-NoDerivatives 4.0 International (CC BY-NC-ND 4.0) License. 